\title{
ECG Anomalies Identification Using a Time Series Novelty Detection Technique
}

\author{
A.P. Lemos ${ }^{1}$, C. J. Tierra-Criollo ${ }^{1}$ and W.M. Caminhas ${ }^{1}$ \\ ${ }^{1}$ Universidade Federal de Minas Gerais/Departamento de Engenharia Elétrica, Belo Horizonte, Brazil
}

\begin{abstract}
This work proposes a methodology for identifying heart anomalies on electrocardiograms using a time series novelty detection technique. Novelties or anomalies on time series can be seen as unexpected values or a sequence of unexpected values when compared to a dataset considered to be normal. The technique uses an autoregressive model to estimate the current value of the time series and compares this value with the observed value, if the difference between these two values exceeds one given threshold; the value is classified as a novelty. To build the thresholds this method uses confidence intervals built upon the probability density function of the forecasting model output. In order to treat anomaly as a sequence of data points a post processing technique is applied to the detector output. Experiments were executed on ECG containing anomalies heart beats and the method was able to detect the anomalies with the false alarm expected.
\end{abstract}

Keywords- Heart ECG anomalies, novelty detection, AR model, confidence interval.

\section{INTRODUCTION}

The normal behavior of a system can be characterized by a series of observations through the time. The problem of novelty detection or anomaly detection consists in finding time periods where some characteristic of the monitored system has been changed. One application of novelty detection can be found on electrocardiogram (ECG) analysis, where the anomaly can be seen as some abnormal ECG rhythm.

The technique uses an autoregressive model to estimate the present value of the time series. Assuming the data series noise as Gaussian, the model output is also Gaussian. Given the distribution parameters, a confidence interval is computed based on a desired false alarm probability. For each new data point to be classified, its forecasting model output distribution and confidence intervals are calculated using past values of the time series. Finally, if the data point is outside the confidence interval it is classified as a novelty.
The abnormal ECG rhythm to be detected is characterized by an arrhythmia [1], and is composed by a sequence of time series points. In that way a detector output processing technique is used to analyze the time series using a window of points.

This work aims to identify abnormal ECG rhythms, like premature ventricular contractions and atrial premature beats using a novelty detection technique. The technique identifies any abnormal ECG rhythm with no prior knowledge about the type of the abnormal rhythm present on the ECG.

This paper is organized as follows. In Section II the technique proposed is described, including the autoregressive forecasting model, the estimator for its parameters, the detector and the detector output processing. In Section III the novelty detection methodology is used to identify abnormal ECG rhythms. Finally, Section IV presents conclusions and suggestions for further research.

\section{MAterial AND METHODS}

\section{A. Forecasting Model}

An autoregressive model of order $p, A R(p)$ estimates the current value of a stochastic process as a linear combination of its last $p$ values and a white noise. The white noise process, $a_{t}$, is assumed to be Gaussian, independent and identically distributed (i.i.d), with zero mean and variance $\sigma_{a}^{2}$. This model can be written as:

$$
\hat{Z}_{t}=\phi_{1} Z_{t-1}+\phi_{2} Z_{t-2}+\ldots+\phi_{p} Z_{t-p}+a_{t}
$$

where $\hat{Z}_{t}$ is the current estimated value and $\phi=\phi_{1}, \phi_{2}, \ldots, \phi_{p}$ are real values.

Given a time series that can be described as a stochastic process, to build a forecasting model for this series using (1) initially it is necessary to estimate the model parameters for 
several values of $\mathrm{p}$ and then to evaluate which is the most suitable value for $\mathrm{p}$ using some statistical criterion.

The Maximum Likelihood Estimator for the model parameters, $\phi=\phi_{1}, \phi_{2}, \ldots, \phi_{p}$ and, $\sigma_{a}^{2}$ is defined as [2] [3]:

$\hat{\phi}=\left(X^{\prime} X\right)^{-1}\left(X^{\prime} Z\right)$

$\hat{\sigma}_{a}^{2}=\frac{1}{N-p}(Z-X \hat{\phi})(Z-X \hat{\phi})^{-1}$

where $Z=\left[\begin{array}{l}Z_{p+1} \\ Z_{p+2} \\ \vdots \\ Z_{N}\end{array}\right]$ and $X=\left[\begin{array}{lll}Z_{p} & \cdots & Z_{1} \\ Z_{p-1} & \cdots & Z_{2} \\ \vdots & \vdots & \vdots \\ Z_{N-1} & \cdots & Z_{N-p}\end{array}\right]$

Once the parameters of several $A R(p)$ models have been calculated, the most suitable value of $p$ must be estimated. The Scharwaz's Bayesian Information Criterion (BIC) [4] is used to select the $\mathrm{p}$ value. The BIC is given by:

$B I C=\log \left(\sigma_{a}^{2}\right)+\frac{M \log (N)}{N}$

where $M=p+1$ and $N$ is the length of the time series.

\section{B. The Detector}

The detector must be capable to distinguish between the following hypotheses:

- $H_{0}-Z_{t}$ is normal

- $H_{1}-Z_{t}$ is a novelty

For a given input $Z_{t}$ the detector must be capable to classify the point as normal meaning that it can be predicted by the forecasting model build for the time series, or novelty, the point can not be predicted by the model.

The detection probability $P_{D}$ is the probability of the detector to classify the point as a novelty correctly, $P\left(\mathrm{H}_{1}\right.$; H1). The false alarm probability $P_{F A}$ is the probability of the detector to classify the point as a novelty when the point is actually normal, $P\left(\mathrm{H}_{1} ; \mathrm{HO}\right)$.

Initially, the forecasting model parameters are estimated using a dataset of the time series assumed as normal. Then, for each new observed value of the time series $Z_{t}$ the statistical inference on the hypotheses is performed by the following steps:

1. The predicted value $\hat{Z}_{t}$ is calculated using $p$ last points of the time series.
2. The parameters of the predicted value distribution are estimated and the thresholds of an $\alpha \%$ confidence interval are calculated.

3. If the observed value of the time series $Z_{t}$ is inside the confidence interval, the null hypothesis is considered true.

The probability density function of the forecasting model output $\hat{Z}_{t}$ when the null hypothesis is true $p\left(\hat{Z}_{t} ; H_{o}\right)$ on a given instant of time $t$ is:

$p\left(\hat{Z}_{t} ; H_{o}\right)=\frac{1}{\sqrt{2 \pi} \hat{\sigma}_{a}^{2}} e^{=\frac{1}{2 \hat{\sigma}_{a}^{2}}\left(\hat{Z}_{t}-\mu_{\hat{z}_{t}}\right)^{2}}$

where $\mu_{\hat{Z}_{t}}=\phi_{1} Z_{t-1}+\cdots+\phi_{p} Z_{t-p}$.

The thresholds of the $\alpha \%$ confidence interval $\left[\gamma_{1}, \gamma_{2}\right]$ are given by:

$\gamma_{1}=\mu_{\hat{z}_{t}}+Q^{-1}\left(\frac{100-\alpha}{200}\right) \hat{\sigma}_{a}^{2}$
$\gamma_{2}=\mu_{\hat{z}_{t}}-Q^{-1}\left(\frac{100-\alpha}{200}\right) \hat{\sigma}_{a}^{2}$

where $Q(x)$ is the complementary cumulative distribution function of the normal distribution.

The value of the confidence interval is calculated given a desired false alarm probability. For example, if it's desired to have a $5 \%$ false alarm probability a $95 \%$ confidence interval must be calculated.

The detection probability is given by:

$P_{D}=P\left(H_{1} ; H_{1}\right)=\operatorname{Pr}\left(\hat{Z}_{t}<\gamma_{1} ; H_{1}\right)+\operatorname{Pr}\left(\hat{Z}_{t}>\gamma_{2} ; H_{1}\right)(6)$

The novelty probability distribution $p\left(\hat{Z}_{t} ; H_{1}\right)$ it is not know, so it is not possible to calculate the detection probability analytically. To estimate this probability is necessary have prior knowledge about the abnormal ECG rhythms to be detected. However this work focuses on identifying the anomalies and not to classifying then.

\section{Detector Output Processing}

The detector presented classifies each point of the time series as a novelty or normal. However, the novelty to be detected, an anomaly heart beat, it is formed by a sequence of points. So, in order to use this detector on an ECG it is proposed a technique to process the detector output using a sliding window of size $W$.

Given $N$ detector outputs related to $N$ data points of the time series, $N-W+1$ windows are generated, in such a way 
that the first window is formed by the outputs on the interval $[1, W]$, the second on the interval $[2, W+1]$, and so on:

$\left[\begin{array}{cccc}c_{1} & c_{2} & \ldots & c_{W} \\ c_{2} & c_{3} & \ldots & c_{W+1} \\ \vdots & \vdots & \ddots & \\ c_{N-W+1} & c_{N-W+2} & \ldots & c_{N}\end{array}\right]$

where $c_{t}$ is the detector output relative to a time series data point $Z_{t}$ on the time instant $t$.

Each window is defined as an event $e_{W}(t)$ and its 1norm is given by:

$\left|e_{w}(t)\right|=\sum_{i=1}^{W} c_{i}$

and measures how many novelties are found on the event.

Assuming that the detector output is an identical distributed Bernoulli variable, the $\left|e_{w}(t)\right|$ probability distribution will be a binomial random variable:

$p_{\left|e_{w}(t)\right|}=\left\{\begin{array}{l}\left(\begin{array}{c}W \\ \left|e_{w}\right|\end{array}\right) p^{\left|e_{w}\right|}(1-p)^{W-\left|e_{w}\right|},\left|e_{w}\right|=0, \ldots, W \\ 0, \text { otherwise }\end{array}\right.$

where $p$ is the probability of occurrence of a novelty, and it is given by $p=1-\alpha / 100$.

A $\alpha \%$ unilateral confidence interval is built upon $p_{\left|e_{w}(t)\right|}$ and if the value of $\left|e_{w}(t)\right|$ is bigger than this interval, all the points present in this event (window $\mathrm{W}$ ) are classified as novelties. The threshold of this confidence interval is calculated finding the smallest integer whose $\left|e_{w}(t)\right|$ cumulative distribution function evaluated in this point is equal or exceeds the value of $\alpha$.

After this procedure, each point of the interval $[W, W$ $N+1]$ will have $W$ distinct classifications. The final result for each point is obtained defining a percentage of novelties classifications that each point must have to be classified as a novelty. This constant is a parameter of the proposed algorithm defined as $k$ and is defined for the interval $(0,100]$.

\section{RESULTS}

The novelty detection technique described above was used to identify abnormal ECG rhythms on two electrocardiograms taken from [5].

Figure 1 illustrates a part of the first ECG annotated by a cardiologist as containing two premature ventricular con- tractions. The anomalous beats were annotated by a "V" and the normal beats where annotated by an "N".

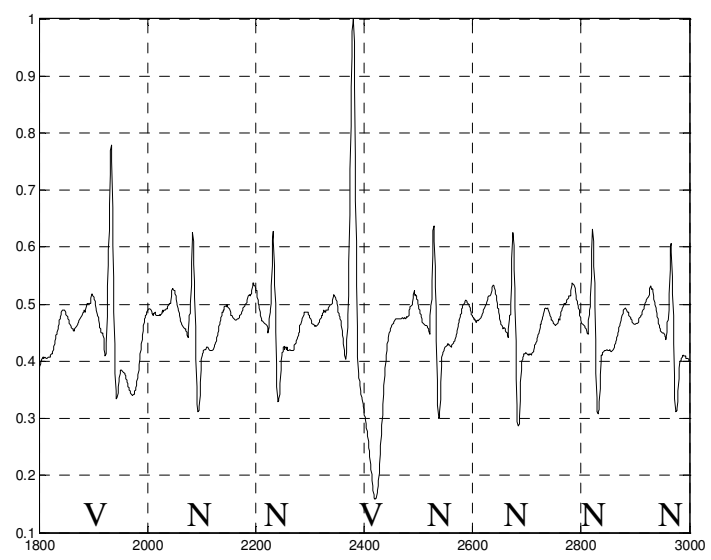

Fig. 1 - ECG containing two premature ventricular contractions (V) and six normal beats $(\mathrm{N})$.

The time series corresponding with this ECG is formed by 3000 data points and the two novelties events to be detected are present in the last 1200 points. The first 1000 points were used to estimate the $A R(p)$ model parameters and the points of the interval [1000,1800] were used to validate the model. Finally the points of the interval [1801, 3000] were used as inputs to be classified.

The parameters of the algorithm were set to $\mathrm{k}=10 \%$, $\mathrm{W}=150$ points (mean R-R interval), $\alpha=95 \%$.. An AR(10) model was used and figure 2 illustrates the final classification of the points, where ' 0 ' is a normal beat and ' 1 ' is one abnormal. Analyzing this figure one can see that the technique proposed was able to detect the two anomalies.

The second electrocardiogram used contains two atrial premature beats to be detected. Figure 3 illustrates the part of the ECG annotated by a cardiologist as containing the anomalies. The anomalous beats were annotated by an "A" and the normal beats where annotated by an "N".

The time series corresponding with this ECG is formed by 2600 data points and the two novelties events to be detected are present in the last 1500 points. The first 800 points were used to estimate the $A R(p)$ parameters and the points of the interval $[801,1100]$ were used to validate the model. Finally the points of the interval $[1101,2600]$ were used as inputs to be classified. 


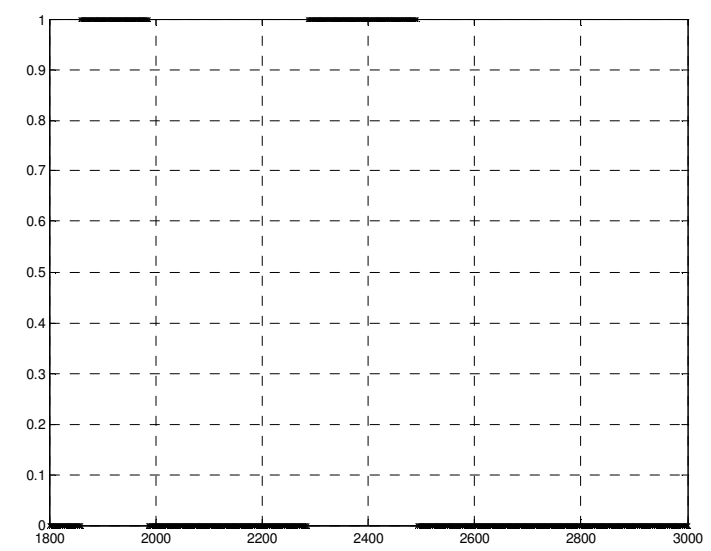

Fig. 2 - Final classification of the data points of the first experiment.

The parameters of the algorithm were set to $k=10 \%$, $W=200$ points (mean $R-R$ interval), $\alpha=95 \%$. An $A R(6)$ model was used and figure 4 illustrates the final classification. Analyzing this figure one can see that the technique proposed was able to detect the two anomalies

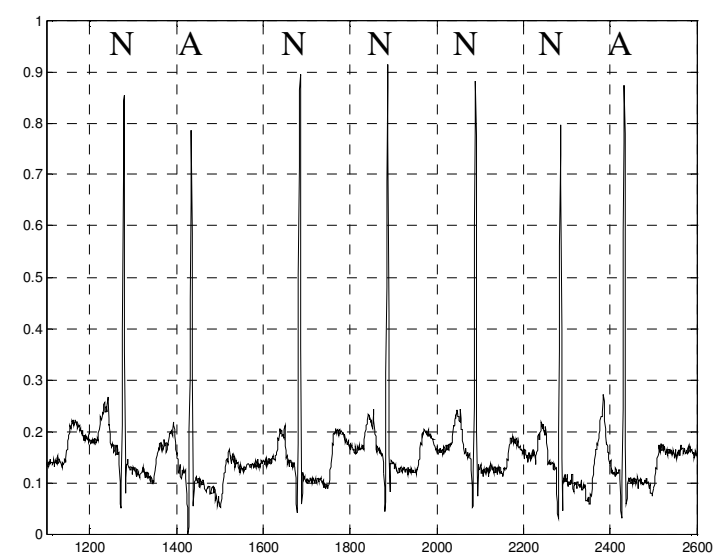

Fig. 3 - ECG containing two atrial premature beats (A) and six normal beats $(\mathrm{N})$.

A final experiment was executed using a 15 minute version of the first experiment ECG containing 19 premature ventricular contractions. The time series corresponding with this ECG contains 225000 points and was differentiated in order to become stationary [2]. The first 3000 points were used to train the model and the next 3000 points were used to validate the model. The algorithm (with the same parameters of the first experiment) detected all the 19 anomalies, with a $5.7 \%$ false alarm rate as expected.

\section{CONCLUSIONS}

This works proposes a methodology to detect heart anomalies based on a novelty detection technique.

The results showed that the technique was able to detect two types of anomalous heart beats on different electrocardiograms.

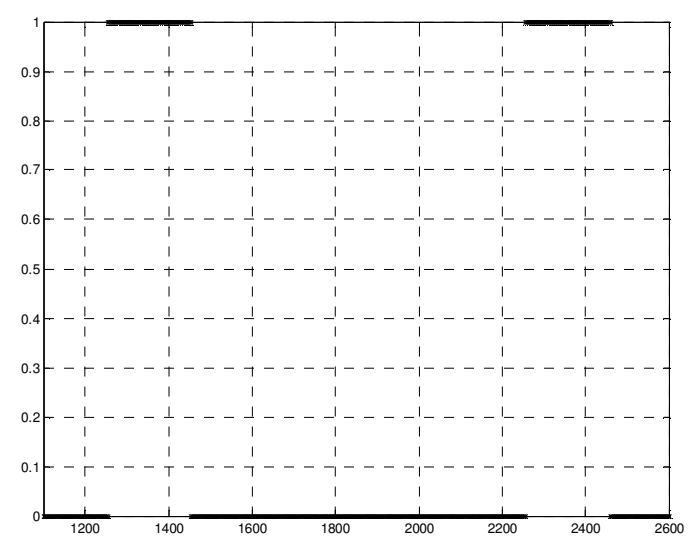

Fig. 4 - Final classification of the data points of the second experiment.

This technique has a limitation of model only stationary time series due the forecasting model proposed. So as suggestions for further work one can extend this methodology using an adaptive $A R(p)$ model, so it can be used on nonstationary time series.

More experiments are being executed in order to evaluate more precisely the detector performance.

\section{REFERENCES}

1. Jenkins, J. M and Jenkins R. E.(2003) Arrhythmia database for algorithm testing: surface leads plus intracardiac leads for validation. Journal of Electrocardiology Vol. 36 Supplement 2003

2. Box, G. E. and Jenkins, G. (1990). Time Series Analysis, Forecasting and Control. Holden-Day, Incorporated

3. Davis, M. and Vinter, R. (1985). Stochastic Modeling and Control. Chapman Hall.

4. Schwarz, G. (1978). Estimating the dimension of a model. The Annals of Statistics 6:461-464

5. P. Laguna, R.G. Mark, A. Goldberger, and G.B. Moody (1997) A database for evaluation of algorithms for measurement of QT and other waveform intervals in the ECG. Comput. Cardiology, vol. 24, pp. 673-676, 1997.

Author: André Paim Lemos

Institute: Universidade Federal de Minas Gerais

Street: $\quad 6627$, Antônio Carlos Avenue

City: Belo Horizonte

Country: Brazil

Email: andrepl@cpdee.ufmg.br 